\title{
Calidad de semillas en colectas y accesiones de Jatropha curcas $\mathbf{L}$.
}

\author{
Seed quality in collections and accessions of Jatropha curcas $L$. \\ Cipriano Ramón Enciso-Garay ${ }^{1 *}$, Francisco Agustín Vergara Ocampo ${ }^{1}$, \\ Victoria Rossmary Santacruz Oviedo ${ }^{2}$
}

\section{RESUMEN}

Esta investigación se llevó a cabo con el propósito de evaluar la calidad de semillas obtenidas de la colección de germoplasma de Jatropha curcas L. del Centro Tecnológico Agropecuario en Paraguay (CETAPAR), ubicado en el distrito de Yguazú, departamento de Alto Paraná, Paraguay. Los tratamientos estuvieron constituidos de materiales provenientes de colectas de las localidades paraguayas: Guayaki, Juan León Mallorquín, Minga Guazú, Caraguatay, La Colmena, Sapucai, Fulgencio R. Moreno, Santa Rosa del Aguaray y Horqueta; además de accesiones del estado brasileño de Minas Gerais, cuyas denominaciones son: Bento, Filomena, Gonzalo, Oracilia y Paraguaçu y de un material del estado de Mato Grosso do Sul, que lleva como nombre Dourados. La calidad de semillas fue evaluada según la masa de mil semillas, vigor, poder germinativo y el contenido de aceite. Los resultados estadísticos muestran que para todas las variables evaluadas existen diferencias entre los tratamientos. Los mayores contenidos de aceite en semillas se presentan en las colectas provenientes de Juan León Mallorquín, Guayaki y La Colmena; mientras que para el vigor, poder germinativo y peso de mil semillas, se destaca la procedente de Fulgencio R. Moreno.

Palabras clave: Jatropha curcas L., germoplasma, contenido de aceite, germinación y vigor de semillas.

\begin{abstract}
This research was conducted in order to assess the quality of seeds obtained from the germplasm collection of Jatropha curcas L. at the Centro Tecnológico Agropecuario en Paraguay (CETAPAR), located in the district of Yguazú, department of Alto Paraná, Paraguay. The treatments consisted of materials collected in Paraguay at the localities of Guayaki, Juan León Mallorquín, Minga Guazu, Caraguatay, La Colmena, Sapucai, Fulgencio R. Moreno, Santa Rosa del Aguaray and Horqueta; as well as accessions from the Brazilian state of Minas Gerais, whose names are Bento, Filomena, Gonzalo, Oracilia and from the state of Mato Grosso do Sul which bears the name of Dourados. Seed quality was evaluated by the average weight of thousand seeds, vigour, germination percentage and oil content. For all evaluated variables, statistical analyzes showed significant differences between treatments. Higher oil contents in seeds were found in the collections from Juan Leon Mallorquin, Guayaki and La Colmena, while Fulgencio $R$. Moreno had the highest vigour, germination and seed weight.
\end{abstract}

Key-word: Jatropha curcas L., germplasm, oil content, seed germination and vigour.

\section{Introducción}

El piñón o jatropa es una especie de origen tropical de la familia Euforbiácea que lleva por nombre científico Jatropha curcas L.; este deriva etimológicamente del griego: játros: doctor, y trophé: alimento. Mientras que curcas es el nombre común que recibe la nuez Phycis en la India (Sotolongo et al., 2007). Existen alrededor de doscientos nombres comunes para esta planta, los cuales indican que $J$. curcas tiene una amplia distribución (NRCS, 2008).
Nativa de México y América Central, es cultivada en regiones tropicales y subtropicales de todo el mundo (Heller, 1996).

En el Paraguay esta especie se encuentra diseminada en forma natural en la región oriental, principalmente en el norte; en los departamentos de Concepción y San Pedro, en el centro, en los departamentos de Cordillera y Paraguarí, en el centro-este, en el departamento de Caaguazú y en el este, en el departamento de Alto Paraná, donde son utilizados como cercas vivas alrededor de las

1 Universidad Nacional de Asunción, Facultad de Ciencias Agrarias. Ruta N², Mcal. Estigarribia, km 11,0. Campus Universitario, San Lorenzo, PY.

2 Instituto Paraguayo de Tecnología Agraria (IPTA).

* Autor por correspondencia: cenciso@agr.una.py

Fecha de Recepción: 04 Marzo, 2013.

Fecha de Aceptación: 16 Abril, 2013. 
viviendas o como sombra. Por la amplia distribución en las diferentes zonas del país se espera que exista una variabilidad genética que puede ser aprovechada en trabajos de mejoramiento genético.

El interés en esta especie surge por el aceite que contienen sus semillas que puede ser transformado en biodiesel mediante el proceso de transesterificación. Las investigaciones realizadas en este ámbito muestran variaciones en el contenido, entre $27,75 \%$ y 40\% (Huerga et al., 2010; Ramesh et al., 2006; Sánchez \& Salinas, 2010; Heller, 1996). Además, como subproductos derivados de la elaboración del aceite se obtienen glicerina y fertilizantes orgánicos.

El piñón se puede propagar en forma vegetativa por estacas, injerto y cultivo de meristemos; sin embargo, la propagación por semillas es la más utilizada para la implantación de cultivos comerciales. Conforme a Saturnino et al. (2005), las plantas provenientes de semillas son robustas y resistentes a la sequía, de mayor longevidad y con un sistema radical con mayor capacidad para explorar el suelo.

De modo general, según Marcos Filho \& Pereira (2006), la literatura documenta la relación existente entre la germinación y el vigor de las semillas, evaluadas en laboratorio y la emergencia de las plántulas en campo. Se conoce que bajo condiciones ambientales adecuadas después de la siembra, los resultados de la germinación se aproximan al porcentaje de emergencia, pero la evaluación del vigor es necesaria para estimar el potencial de desempeño de las semillas cuando las condiciones del ambiente se desvían de las más adecuadas.

El uso de semillas con calidad es el principal requisito para que la semilla pueda ser utilizada con éxito en el establecimiento de un cultivo (Marcos Filho, 2005).

Considerando el interés existente en el piñón como potencial para la industria de biocombustibles y la carencia de información básica sobre el cultivo, esta investigación se llevó a cabo con el propósito de evaluar la calidad de semillas de colectas y accesiones de piñón provenientes de Paraguay y Brasil, en términos de masa de mil semillas, porcentaje de germinación, vigor y contenido de aceite.

\section{Materiales y Métodos}

La evaluación se realizó entre agosto y septiembre de 2009 con semillas obtenidas de la colección de germoplasma de piñón del Centro Tecnológico Agropecuario en Paraguay (CETAPAR), dependiente de la Agencia de Cooperación Internacional del Japón (JICA), ubicada a 281 kilómetros de la ciudad de Asunción, sobre la Ruta VII, "Dr. José Gaspar Rodríguez de Francia", en el distrito de Yguazú, departamento de Alto Paraná, Paraguay. Las coordenadas geográficas son: $25^{\circ} 27^{\prime} 20^{\prime \prime}$ latitud sur y $55^{\circ} 02$ ' $36^{\prime \prime}$ longitud oeste.

El suelo de la zona donde se instaló el experimento corresponde al orden Oxisol, presenta contenido de arcilla de 2,33\% y pH de 6,63.

Los genotipos paraguayos corresponden a colectas realizadas en las localidades que tienen el mismo nombre; mientras que los provenientes del Brasil a excepción de Dourados que proviene del estado de Mato Grosso do Sul, las demás corresponden a accesiones de la empresa NNE Minas Agroflorestal, ubicada en el estado de Minas Gerais (Tabla 1).

La cosecha de los frutos maduros para las evaluaciones se realizó en julio de 2009, procedente de parcelas implantadas en agosto de 2007, con un arreglo espacial de $3 \mathrm{~m}$ entre hileras y $2 \mathrm{~m}$ entre plantas. Los frutos cosechados fueron llevados a una estufa por tres días a $80{ }^{\circ} \mathrm{C}$ para el secado y posteriormente se realizó el desgranado en forma manual.

Las evaluaciones se realizaron en los laboratorios del Centro Tecnológico Agropecuario en Paraguay y fueron las siguientes:

- Masa media de mil semillas: se calculó multiplicando por diez el peso obtenido de las mediciones llevadas a cabo con cien semillas; fueron cuatro repeticiones por cada tratamiento. Dichas mediciones se efectuaron con una balanza de precisión.

- Germinación y vigor de semillas: para determinar el poder germinativo y vigor de las semillas se sembraron tres repeticiones de 50 semillas por tratamiento, en bandejas conteniendo arena lavada, previamente esterilizada. Luego de la siembra, las bandejas se colocaron en el germinador a una temperatura de $\pm 28^{\circ} \mathrm{C}$. Las evaluaciones se realizaron de acuerdo con el método ISTA (2005); a los siete días de la siembra, para el caso del vigor; y a los 14 días para el poder germinativo.

- Contenido de aceite en semillas: la cuantificación del contenido de aceite en las semillas se determinó gravimétricamente después de la extracción de las muestras con hexano caliente, en extractor Soxhlet; para ello se tomaron tres 
Tabla 1. Colectas y accesiones de piñón (Jatropha curcas) evaluados. CETAPAR, Yguazú, PY, 2009.

\begin{tabular}{cllr}
\hline Tratamiento & Lugar de procedencia & Departamento/estado & País \\
\hline 1 & Guayaki & Caaguazú & Paraguay \\
2 & Juan L. Mallorquín & Alto Paraná & Paraguay \\
3 & Minga Guazú & Alto Paraná & Paraguay \\
4 & Caraguatay & Cordillera & Paraguay \\
5 & La Colmena & Paraguari & Paraguay \\
6 & Sapucai & Paraguari & Paraguay \\
7 & Fulgencio R. Moreno & Alto Paraná & Paraguay \\
8 & Santa Rosa del Aguaray & San Pedro & Paraguay \\
9 & Horqueta & Concepción & Paraguay \\
10 & Dourados & Mato Grosso do Sul & Brasil \\
11 & Bento & Minas Gerais & Brasil \\
12 & Filomena & Minas Gerais & Brasil \\
13 & Gonçalo & Minas Gerais & Brasil \\
14 & Oracilia & Minas Gerais & Brasil \\
15 & Paraguaçu & Minas Gerais & Brasil \\
\hline
\end{tabular}

muestras de 50 gramos por cada lote de semillas, obteniendo de este modo tres repeticiones por cada tratamiento.

El diseño experimental utilizado para todas las evaluaciones fue completamente al azar. Los datos obtenidos en las mediciones fueron sometidos a análisis de varianza y en los casos en que se obtuvieron diferencias significativas, las medias fueron comparadas entre sí, por la prueba de Tukey al 5\% de probabilidad.

\section{Resultados y Discusión}

El análisis de los resultados de la masa de mil semillas presentó diferencias estadísticas significativas en función a los materiales genéticos evaluados (Tabla 2). El material procedente de Fulgencio R. Moreno fue el que generó la mayor media, difiriendo estadísticamente, apenas, de los tratamientos Juan León Mallorquín, La Colmena y Caraguatay. Los que obtuvieron los menores valores para esta variable fueron Bento, Gonçalo y Oracilia, los cuales no difirieron significativamente a su vez del tratamiento Filomena. Las diferencias encontradas en la masa de mil semillas se deben posiblemente a la variabilidad genética existente entre los materiales evaluados, teniendo en cuenta que todos ellos recibieron los mismos cuidados y estuvieron en las mismas condiciones. Estos resultados presentan similitud con los de Ginwal et al. (2005) que en caracterizaciones de semilla de piñón observaron que la masa de las semillas varía dependiendo del origen del material genético.
Por otra parte, Montes et al. (2011) no detectaron diferencias en la masa de semillas de piñón provenientes de diferentes países.

Los valores obtenidos en este estudio para la masa de mil semillas, que oscilaron entre 737,0 g y $607,6 \mathrm{~g}$, se encuentran en el rango reportado por Fogaça et al. (2007) y Höring et al. (2011) quienes citan valores entre 721,5 y 657,6 g. Sin embargo, son superiores a lo relatado por Silva et al. (2008) que encontraron una media de 468,9 g.

En cuanto a vigor de semillas, a través de la comparación de medias por la prueba de Tukey al $5 \%$, se pudo constatar que el material procedente de Fulgencio R. Moreno generó la mayor media $(90,0 \%)$, difiriendo estadísticamente con los tratamientos Guayaki, Minga Guazú, Caraguatay, La Colmena, Dourados, Bento, Filomena, Gonçalo y Paraguaçu. El material proveniente de Caraguatay $(52,0 \%)$ presentó el menor valor, pero sin diferir de los materiales Gonçalo, Bento, La Colmena, Minga Guazú y Guayaki (Tabla 2).

Para el vigor de las semillas de piñón, investigaciones realizadas por Ginwal et al. (2005) reportaron diferencias estadísticas al evaluar semillas procedentes de diferentes materiales genéticos.

Respecto del poder germinativo de las semillas de los diferentes materiales (Tabla 2), el tratamiento Fulgencio R. Moreno $(90,7 \%)$ generó el mayor valor, sin diferir de los tratamientos Juan León Mallorquín, Sapucai, Santa Rosa del Aguaray, Horqueta, Oracilia y Paraguaçu; sin embargo, fue superior estadísticamente de los materiales provenientes de las localidades de Guayaki, Minga 
Tabla 2. Peso de mil semillas (gramos), vigor (porcentaje), poder germinativo (porcentaje) y contenido de aceite de piñón (Jatropha curcas) en los diferentes materiales. CETAPAR, Yguazú, PY, 2009.

\begin{tabular}{|c|c|c|c|c|}
\hline $\begin{array}{l}\text { Tratamientos } \\
\text { (T) }\end{array}$ & $\begin{array}{l}\text { Peso de mil } \\
\text { semillas } \\
(\mathrm{g})\end{array}$ & $\begin{array}{l}\text { Vigor } \\
(\%)\end{array}$ & $\begin{array}{l}\text { Poder germinativo } \\
\qquad(\%)\end{array}$ & $\begin{array}{l}\text { Contenido de aceite } \\
\qquad(\%)\end{array}$ \\
\hline T1: Guayaki, PY & $665,0 \mathrm{de}$ & 63,3 cdef & $71,3 \mathrm{bcd}$ & $34,2 \mathrm{a}$ \\
\hline T2: Juan León Mallorquín, PY & $726,0 \mathrm{ab}$ & $78,0 \mathrm{ab}$ & $82,0 \mathrm{ab}$ & $34,4 \mathrm{a}$ \\
\hline T3: Minga Guazú, PY & $686,3 \mathrm{~cd}$ & 60,6 def & $62,7 \mathrm{~cd}$ & $32,0 \mathrm{c}$ \\
\hline T4: Caraguatay, PY & $714,3 \mathrm{abc}$ & $52,0 \mathrm{f}$ & $58,0 \mathrm{c}$ & $32,7 \mathrm{~b}$ \\
\hline T5: La Colmena, PY & $715,6 \mathrm{abc}$ & 58,0 ef & $60,7 \mathrm{~d}$ & $34,5 \mathrm{a}$ \\
\hline T6: Sapucai, PY & $693,0 \mathrm{bcd}$ & $78,0 \mathrm{ab}$ & $81,3 \mathrm{ab}$ & $32,6 \mathrm{~b}$ \\
\hline T7: Fulgencio R. Moreno, PY & $737,0 \mathrm{a}$ & $90,0 \mathrm{a}$ & 90,7 a & $31,5 \mathrm{~d}$ \\
\hline T8: Sta. Rosa del Aguaray, PY & $670,3 \mathrm{~d}$ & $81,3 \mathrm{ab}$ & $82,7 \mathrm{ab}$ & $31,3 \mathrm{~d}$ \\
\hline T9: Horqueta, PY & 664,6 de & $81,3 \mathrm{ab}$ & $83,3 \mathrm{ab}$ & $31,4 \mathrm{~d}$ \\
\hline T10: Dourados, BR & $663,6 \mathrm{de}$ & $72,0 \mathrm{bcd}$ & $72,7 \mathrm{bcd}$ & $31,7 \mathrm{~cd}$ \\
\hline T11: Bento, BR & $617,3 \mathrm{f}$ & $62,0 \mathrm{def}$ & $62,0 \mathrm{~cd}$ & $30,3 \mathrm{e}$ \\
\hline T12: Filomena, BR & 633,3 ef & 70,0 bcde & $70,0 \mathrm{bcd}$ & $28,7 \mathrm{f}$ \\
\hline T13: Gonçalo, BR & $622,3 \mathrm{f}$ & 58,0 ef & $59,3 \mathrm{~d}$ & $30,0 \mathrm{e}$ \\
\hline T14: Oracilia, BR & $607,6 \mathrm{f}$ & $79,3 \mathrm{ab}$ & $84,7 \mathrm{ab}$ & $26,2 \mathrm{~g}$ \\
\hline T15: Paraguaçu, BR & $672,0 \mathrm{~d}$ & $75,3 \mathrm{bc}$ & $76,7 \mathrm{abc}$ & $30,1 \mathrm{e}$ \\
\hline Promedio & 672,58 & 70,62 & 73,20 & 31,47 \\
\hline Coeficiente de variación (\%) & 1,82 & 6,03 & 7,18 & 0,46 \\
\hline
\end{tabular}

Medias seguidas de la misma letra en las columnas, no difieren estadísticamente por la prueba de Tukey al 5\% de probabilidad. PY: Material paraguayo. BR: Material brasileño.

Guazú, Caraguatay, La Colmena, Dourados, Bento, Filomena y Gonçalo, verificándose que éste último presentó el menor poder germinativo $(59,3 \%)$. Estos resultados son concordantes con Ginwal et al. (2005), los que relatan la existencia de diferencias estadísticas en el poder germinativo de las semillas de piñon en función a la procedencia del material genético, dichos autores reportaron valores entre $61,2 \%$ y $85,7 \%$ de germinación.

El hecho de que el material procedente de Fulgencio R. Moreno haya presentado las mayores medias para el vigor y poder germinativo puede ser debido a que también presentó la mayor masa de mil semillas. Conforme a Carvalho \& Nakagawa (2000) las semillas de mayor tamaño fueron mejor nutridas durante su desarrollo, contienen embriones bien formados y con mayor cantidad de sustancias de reserva, siendo consecuentemente más vigorosas.

La capacidad de germinar y formar una plántula sana y normal es el principal factor para determinar la calidad y el potencial de las semillas.

En cuanto al contenido de aceite, los materiales procedentes de Guayaki, Juan L. Mallorquín y La Colmena proporcionaron las medias más elevadas, con $34,2 \%, 34,4 \%$ y $34,5 \%$, respectivamente, presentando diferencias significativas con los demás tratamientos; en segundo orden se ubicaron los materiales procedentes de Caraguatay $(32,7 \%)$ y Sapucai $(32,6 \%)$, estadísticamente similares entre ambos. El tratamiento Oracilia fue el que mostró menor porcentaje de aceite en sus semillas $(26,2 \%)$, difiriendo estadísticamente con los demás materiales evaluados (Tabla 2).

Esto concuerda con las investigaciones realizadas por Ginwal et al. (2004) quienes al evaluar el contenido de aceite en semillas, encontraron diferencias estadísticas entre los materiales, donde los contenidos de aceite estuvieron entre 33,0 y $39,1 \%$, dichos autores mencionan que esas diferencias se deben a la variabilidad genética existente en los materiales evaluados. También Rocha et al. (2012) observaron diferencias significativas en el contenido de aceite en las semillas, obteniendo una media general de $32,0 \%$, mientras que en este experimento la media fue de $31,47 \%$.

Los valores obtenidos en este experimento, que oscilaron entre $26,2 \%$ y $34,5 \%$ de aceite, son próximos a lo presentado por Oliveira et al. (2008) quienes al evaluar semillas provenientes de plantas 
silvestres de piñón encontraron $31,6 \%$ de aceite y por Huerga et al. (2010) que reportaron amplitudes entre $25,75 \pm 2,05$ y $32,00 \pm 1,7 \%$. Sin embargo, las medias encontradas en este experimento son inferiores a lo señalado por Sánchez \& Salinas (2010), quienes citan contenidos de aceite entre 37,0 y $38,0 \%$.

Debido a que la importancia económica del piñón radica fundamentalmente en su capacidad de producir aceite, la selección de aquellos materiales genéticos con mayor contenido oleoso, posibilitará un aumento en los rendimientos industriales; y si en el futuro se toma en cuenta esta característica para la cotización comercial de las semillas, significará también un aumento en los ingresos del productor.

\section{Conclusiones}

1. Existen diferencias en la calidad de semillas de los materiales genéticos evaluados.

2. En cuanto al vigor, poder germinativo y peso de mil semillas, se destaca la colecta procedente de Fulgencio R. Moreno.

3. Los mayores contenidos de aceite en semillas se presentan en las colectas denominadas Juan León Mallorquín, Guayaki y La Colmena.

\section{Agradecimientos}

Al Centro Tecnológico Agropecuario en Paraguay (CETAPAR), por las facilidades brindadas.

\section{Literatura Citada}

Carvalho, N.M.; Nakagawa, J.

2000. Sementes: ciência, tecnologia e produção. Jaboticabal: FUNEP, $588 \mathrm{p}$.

Fogaça, C.A.; Silva, L.L; Polidoro, J.C.; Breier, T.B.; Leles, P.S.S. 2007. Metodologia para a condução do teste de germinação em sementes de Jatropha curcas L. In: Congresso Brasileiro de Plantas Oleaginosas, Óleos, Gorduras e Biodiese L, 4., Varginha. Anais. Varginha: UFL, 2007, pp. 1351-1357.

Ginwal, H.S.; Rawat, P.S.; Srivastava, R.L.

2004. Seed source variation in growth performance and oil yield of Jatropha curcas Linn in Central India. Silvae Genetica, 53: 186-192.

Ginwal, H.S.; Phartyal, S.S.; Rawat, P.S; Srivastava, R.L. 2005. Seed source variation in morphology, germination and seedling growth of Jatropha curcas Linn in central India. Silvae Genetica, 54: 76-80.

Heller, J.

1996. Physic nut. Jatropha curcas L. Promoting the conservation and use of underutilized and neglected crops. Rome: International Plant Genetic Resources Institute, 66 p.

Höring, C.F.; Malavasi, M.M.; Malavasi, U.C.

2011. Armazenamento não controlado na qualidade de sementes de Jatropha curcas L. Semina: Ciências Agrárias, 32: 521-526.

Huerga, I.; Carrizo, A; Brizuela, G.; Querini, C.

2010 Contenido y calidad de aceite obtenido de semillas de Jatropha curcas cosechadas en Argentina. INTA: Boletín informativo (INTA, documentos), http://inta.gob.ar/documentos/biodiesel-con-aceite-de-jatropha/at_multi_download/ file/IIR-BC-JC-02-0_Biodiesel_con_Aceite_de_Jatropha. pdf. Consultado: 08/09/2012.

ISTA. International Seed Testing Association, Activity Report. 2005. http://www.seedtest.org/upload/cms/user/03Int-MD200603ActivityReport20051.pdf. Consultado: 26/03/ 2012. Marcos Filho, J.

2005. Fisiologia de sementes de plantas cultivadas. Piracicaba: FEALQ, São Paulo. 495 p.

Marcos Filho, J.; Pereira Kikuti, A.L.

2006. Vigor de sementes de rabanete e desempenho de plantas em campo. Revista Brasileira de Sementes, 28: 44-51.
Montes, J.M; Rodríguez Aliciardi, M.J.; Vaca Chávez, J.C.; Guzmán, C; Calandri, E.

2011. Characterization of Jatropha curcas L. seed and its oil, from Argentina and Paraguay. The Journal of the Argentine Chemical Society, 98: 1-9.

NRCS. Natural Resources Conservation Service: Plants Database: Plants profile. USDA: Washington D.C. 2008. http:// plants.usda.gov/java/profile?symbol=JACU2. Consultado: $12 / 03 / 2008$.

Oliveira, J.; Leite, M.; de Souza, B.; Mello, M;. Silva, C.; Rubim, J.; Meneghetti, S.; Suárez, P.

2008. Characteristics and composition of Jatropha gossypiifolia and Jatropha curcas L. oils and application for biodiesel production. Biomass and Bioenergy, 33: 449-453.

Ramesh, D.; Samapathrajan, A.; Venkatachalam, P. 2006. Production of biodiesel from Jatropha curcas oil by using pilot biodiesel plant. The Jatropha Journal, 18: 1-6.

Rocha, R.B.; Ramalho, A.R.; Teixeira, A.L.; Laviola, B.G.;

Silva, F.C.G. da; Militão, J.S.L.T.

2012. Eficiência da seleção para incremento do teor de óleo do pinhão-manso. Pesquisa Agropecuária Brasileira, 47: 44-5.

Sánchez, E.A.P.; Salinas, D.G.C.

2010. Caracterización morfofisiológica de Jatropha curcas L. variedad Brasil cultivada en dos zonas de Colombia. Acta Agronómica, 59: 30-36.

Saturnino, H.M.; Pacheco, D.; Kakida, J.; Tominaga, N.; Gonçalves, N.P.

2005. Cultura do Pinhão Manso (Jatropha curcas L.). Belo Horizonte, Informe Agropecuário, 26: 44-78.

Silva, H.P.; Neves, J.M.G.; Brandão Junior, D.S.; Costa, C.A. 2008. Quantidade de água do substrato na germinação e vigor de sementes de pinhão manso. Revista Caatinga, 21: 178-184.

Sotolongo, J.A.P.; Diaz, A.A.G.; Oca, S.M.L.; Valle, Y.A.; García, S.P.

2007. Potencialidades energéticas y medio ambientales del árbol Jatropha curcas L. en las condiciones edafoclimáticas de la región semiárida de la provincia de Guantánamo. Tecnología Química, 27: 76-82. 
